\title{
Multi-locus sequence types of Acinetobacter baumanii clinical isolates from India
}

\author{
Dabet Rynga ${ }^{1}$, Malini Shariff ${ }^{1}$, Monorama Deb $^{2}$ \\ ${ }^{1}$ Department of Microbiology, Vallabhbhai Patel Chest Institute, Delhi University, Delhi, India \\ ${ }^{2}$ Department of Microbiology, Vardhaman Mahavir Medical College and Safdarjung Hospital, New Delhi, India
}

Key words: Acinetobacter baumanii; MLST; ST; India

J Infect Dev Ctries 2013; 7(4):358-360. doi:10.3855/jidc.2827

(Received 02 July 2012 - Accepted 07 August 2012)

Copyright $(C 2013$ Rynga et al. This is an open-access article distributed under the Creative Commons Attribution License, which permits unrestricted use, distribution, and reproduction in any medium, provided the original work is properly cited.

\section{Introduction}

Acinetobacter spp. have emerged as important nosocomial pathogens worldwide. Most Acinetobacter spp are multidrug resistant [1] and able to persist in the hospital environment thus causing serious hospitalacquired infections. Typing methods can distinguish outbreak strains from epidemiologically unrelated strains thus facilitating the institution of infection control measures. Phenotypic typing systems have largely been replaced by a number of molecular typing methods, namely ribotyping, pulsed field gel electrophoresis (PFGE), amplified fragment length polymorphisim (AFLP) analysis, random amplification of polymorphic DNA (RAPD), among others [2]. However, inter-laboratory exchange of data for global epidemiological analysis is not possible with these methods. Multi locus sequence typing (MLST) alleviates this problem by offering the possibility of inter-laboratory transfer of data and comparison of results via the internet. MLST is a highly discriminative method for typing microorganisms and has been applied successfully for the epidemiologic characterization of a variety of bacterial pathogens. The Acinetobacter baumannii MLST Databases hosted at the Department of Zoology, University of Oxford website (http://pubmlst.org/abaumannii/) contain 596 Acinetobacter baumanii isolates from all over the world. However, Indian data on sequence types are not available. The aim of the present study was to type clinical isolates of Acinetobacter baumanii collected from hospitals in Delhi, India and compare them with isolates across the globe using MLST.

\section{The study}

Acinetobacter baumanii isolates from various clinical samples (tracheal aspirate, pus swab, burn wound swab, sputum, drain fluid, and high vaginal swab) obtained from two hospitals in Delhi during 2011 were included in this study. Species were identified using the API 20NE system (BioMérieux, Marcy-l'Etoile, France) and results were confirmed using amplified ribosomal DNA restriction analysis (ARDRA). Isolates were characterized with respect to antibiotic sensitivity patterns, production of betalactamases, and typed using RAPD (data not shown). Twenty-three isolates representing different clones by RAPD and/or different hospitals were further subjected to MLST. Seven housekeeping genes were amplified using the method described by Bartual et al. [3], with some modifications in the sequence of $g y r B$ and $r p o D$ primers as given by Park et al. [4]. PCR products were resolved by agarose gel electrophoresis. Amplified fragments were sequenced by outsourcing to a commercial company. The sequences obtained were trimmed using the Bio Edit Sequence alignment editor software (Ibis Biosciences, Carlsbad, CA, USA). The assignment of alleles and sequence types was performed by the software available in the Department of Zoology, Oxford University website noted above. The sequence types not present in the database were submitted to the website and assigned new ST types.

Out of the 23 isolates tested, 12 belonged to known STs such as ST110 $(\mathrm{n}=2)$, ST188 $(\mathrm{n}=3)$, ST146 (n= 2), ST69 (n= 2), ST103 (n=1), ST108 (n $=1)$ and ST194 $(\mathrm{n}=1)$. STs of eleven isolates were found to be new and were submitted to the PUBMLST 
site for assigning new ST types. All but one were assigned new STs (Table). Isolates within the same ST showed differences in the type of specimen or the hospital from which they were isolated. The PUBMLST database has 596 Acinetobacter baumanii isolates with 391 STs. eBURST analysis of the data (15 March 2012), showed 20 groups (each having 6 or more loci in common) consisting of 137 STs. The remaining STs were all singletons. Among the 20 groups, a founder could be identified in only 8, suggesting great diversity among the isolates. ST92 was the most common ST with 77 isolates distributed in several countries with predominance in China $(n=32)$ and Australia $(\mathrm{n}=30)$. This ST emerged around 2002, and was until recently seen in Korea. Another major ST, ST75 with 23 isolates, was restricted to China and has replaced ST92, which was prevalent earlier in China [6]. However, these STs were not encountered in the present study. Among the STs encountered in the present study, ST146 was found in Korea (2008); ST110 in Argentina (1997) and Korea (2008); ST69 in Australia (1998-2003) and Korea (2003); ST103 in Argentina (1991-2004), Norway (2009) and Portugal (2003); ST194 in Norway (2009); ST108 in Argentina (2005); and ST188 in two isolates from Japan [5]. Hence known STs found in the present study are distributed in different continents of the world.

ST types encountered in the present study were compared with antibiotic resistance patterns. It was observed that isolate with ST146 was extensively drug resistant (XDR), sensitive to both tigecycline and colistin. Contrarily, the ST69 isolate was XDR but resistant to tigecycline and sensitive to colistin. ST110 clone consisted of two isolates which had different antibiotic susceptibilities, even though they produced the same type of beta-lactamase, i.e., AmpC and MBL,

Table. Sequence types of Acinetobacter baumanii isolates encountered in the study

\begin{tabular}{|c|c|c|c|c|c|c|c|c|l|}
\hline $\begin{array}{c}\text { S. } \\
\text { No. }\end{array}$ & ST & gltA & gyrB & gdhB & recA & cpn60 & gpi & rpoD & \multicolumn{1}{|c|}{$\begin{array}{c}\text { Strain submitted to } \\
\text { PubMLST database }\end{array}$} \\
\hline 1 & $368^{*}$ & 31 & 33 & 67 & 40 & 1 & 102 & 7 & In- S 58938 \\
2 & $387^{*}$ & 1 & 15 & 4 & 11 & 4 & 140 & 4 & In- S 95167 \\
3 & $388^{*}$ & 1 & 15 & 4 & 11 & 4 & 95 & 4 & In- S 91318 \\
4 & $389^{*}$ & 1 & 7 & 8 & 11 & 1 & 4 & 14 & In- S 93192 \\
5 & $390^{*}$ & 31 & 33 & 67 & 40 & 1 & 58 & 7 & In- S 99665 \\
6 & $390^{*}$ & 31 & 33 & 67 & 40 & 1 & 58 & 7 & In- S 100924 \\
7 & $391^{*}$ & 1 & 15 & 13 & 12 & 4 & 102 & 2 & In- V 1687/11 \\
8 & $391^{*}$ & 1 & 15 & 13 & 12 & 4 & 102 & 2 & In- V 323/05 \\
9 & $391^{*}$ & 1 & 15 & 13 & 12 & 4 & 102 & 2 & In- V 5930/11 \\
10 & $390^{*}$ & 31 & 33 & 67 & 40 & 1 & 58 & 7 & In- V 5290/10 \\
11 & A & 1 & 10 & 10 & 6 & 1 & 4 & 14 & In- S 84570 \\
& & & & & & & & & \\
12 & 103 & 12 & 17 & 12 & 1 & 29 & 3 & 39 & In- S 90868 \\
13 & 69 & 1 & 46 & 3 & 2 & 2 & 58 & 3 & In- S 98632 \\
14 & 69 & 1 & 46 & 3 & 2 & 2 & 58 & 3 & In- S 101149 \\
15 & 108 & 10 & 12 & 4 & 6 & 4 & 9 & 5 & In- S 101767 \\
16 & 194 & 1 & 15 & 4 & 11 & 4 & 58 & 4 & In- S 102704 \\
17 & 110 & 1 & 15 & 2 & 28 & 1 & 52 & 32 & In- V 7254/10 \\
18 & 110 & 1 & 15 & 2 & 28 & 1 & 52 & 32 & In- V 5290/08 \\
19 & 188 & 31 & 33 & 67 & 40 & 16 & 58 & 7 & In- V 7362/07 \\
20 & 188 & 31 & 33 & 67 & 40 & 16 & 58 & 7 & In- V 3507/06 \\
21 & 188 & 31 & 33 & 67 & 40 & 16 & 58 & 7 & In- S 88877 \\
22 & 146 & 1 & 15 & 13 & 12 & 4 & 14 & 2 & In- V 8095/10 \\
23 & 146 & 1 & 15 & 13 & 12 & 4 & 14 & 2 & In- S 92729 \\
\hline
\end{tabular}

* - New STs encountered in the present study

A - To be assigned

S. No. - Series number 
while the ST188 clone with three isolates showed similar antibiotic susceptibility but differed in the type of beta-lactamase produced (data not shown). The discordance between the ST types and antibiotic resistance and/or beta lactamase production could be explained by the fact that MLST is based on housekeeping genes, but the genes responsible for resistance and virulence could be located on mobile elements and clustered in genomic islands and hence are not linked to particular sequence types [6]. Since the MLST database does not give the sensitivity patterns of most isolates, sensitivity data of the known STs from the present study could not be compared with those throughout the world.

The MLST database does not contain Indian isolates other than those of the present study. Therefore, the prevalence of the ST types in India could not be ascertained. Thus further studies are required to characterize isolates from different parts of the country.

\section{References}

1. Kempf M and Rolain JM (2012) Emergence of resistance to carbapenems in Acinetobacterbaumannii in Europe: clinical impact and therapeutic options. Int J Antimicrob Agents 39:105-114.

2. Peleg AY, Seifert H, Paterson DL (2008) Acinetobacter baumannii: Emergence of a successful pathogen. Clin Microbiol Rev 21: 538-582.
3. Bartual SG, Seifert H, Hippler C, Domínguez Luzon MA, Wisplinghoff H, Rodríguez-Valera F (2005) Development of a multilocus sequence typing scheme for characterization of clinical isolates of Acinetobacter baumannii. J Clin Microbiol 43: 43824390.

4. Park YK, Jung SI, Park KH, Cheong HS, Peck KR, Song JH, Ko $\mathrm{KS}(2009)$ Independent emergence of colistin-resistant Acinetobacter spp. isolates from Korea. Diagn Microbiol Infect Dis 64:43-51.

5. Endo S, Yano H, Hirakata Y, Arai K, Kanamori H, Ogawa M, Shimojima M, IshibashiN, Aoyagi T, Hatta M, Yamada M, Tokuda K, Kitagawa M, Kunishima H, KakuM (2012) Molecular epidemiology of carbapenems-non-susceptible Acinetobacter baumannii in Japan. J Antimicrob Chemother 67: 1623-1626.

6. Dijkshoorn L, Nemec A, Seifert H (2007) An increasing threat in hospitals: multidrug-resistant Acinetobacter baumannii. Nat Rev Microbiol5:939-951.

\section{Corresponding author}

Dr. Malini Shariff

Associate Professor

Department of Microbiology

Vallabhbhai Patel Chest Institute

Delhi University

Delhi 110007

India

Telephone: +91-11-27402424.

Fax: +91-11-27666549.

Email: malini.shariff@gmail.com

Conflict of interests: No conflict of interests is declared. 